\title{
Holographic Cosmological Models on the Braneworld
}

\author{
Samuel Lepe and Joel Saavedra \\ Instituto de Física, Pontificia Universidad Católica de Valparaíso, Casilla 4950, Valparaíso, Chile. \\ Francisco Peña \\ Departamento de Ciencias Fúsicas, Facultad de Ingeniería, Ciencias y Administración, \\ Universidad de la Frontera, Avda. Francisco Salazar 01145, Casilla 54-D, Temuco, Chile.
}

\begin{abstract}
In this article we have studied a closed universe which a holographic energy on the brane whose energy density is described by $\rho(H)=3 c^{2} H^{2}$ and we obtain an equation for the Hubble parameter, this equation gave us different physical behavior depending if $c^{2}>1$ or $c^{2}<1$ against of the sign of the brane tension.

PACS numbers:
\end{abstract}

\section{INTRODUCTION}

From a modern point of view, we have two main ingredient in order to deal with the current problems of modern cosmology (late acceleration of the universe, cosmological constant problem, etc.). One of this element is the inclusion of extra dimension by means of the warped fifth dimension that were provided a new scheme to solve the hierarchy problem in particle physics [1, 2]. These models allows to consider the origin of dark energy (the density matter useful to explain the late acceleration of the universe) from gravitational theory and changing the standard stress tensor for matter [3]

$$
G_{\mu \nu}=8 \pi G_{N}\left(T_{\mu \nu}^{\text {matter }}+T_{\mu \nu}^{\text {dark-energy }}\right),
$$

where $G_{\mu \nu}$ is the $4 D$ Einstein tensor, $T_{\mu \nu}^{\text {matter }}$ is the stress tensor for matter and $T_{\mu \nu}^{\text {dark-energy }}$ is the stress tensor for dark energy, a new exotic component that have a negative pressure to match with the observation. We can modify this description to one more fundamental, where the matching with the observation is arises from the gravitational sector,

$$
G_{\mu \nu}-\mathcal{K}_{\mu \nu}=8 \pi G_{N} T_{\mu \nu}^{\text {matter }},
$$

where $\mathcal{K}_{\mu \nu}$ denotes a tensor that arise from the extrinsic curvature, due to the embedding of our brane universe in the $5 D$ bulk. Therefore from the stadium of cosmologist we can write the modify Friedmann equation as follow,

$$
H^{2}=\frac{8 \pi G_{N}}{3} f(\rho),
$$

where the function $f(\rho)$ encoded all geometric modification of the cosmological equation. In particular the RandallSundrum scenario has gotten a great attention in the last decade [1, 2]. From the cosmological point of view, brane world offers a novel approach to our understanding of the evolution of the universe. One of the most spectacular consequence of this scenario is the modification of the Friedmann equation. In these models, for instance in five dimensions, matter is confined to a four dimensional brane, while gravity can be propagated in the bulk, and can feel the extra dimension. From the perspective of string theory [4], brane world cosmology has been a big challenge for modern cosmology . For a review on BW cosmology see Ref. [5] and reference therein.

The other main ingredient is the holographic principle, that in simple words establish that all degrees of freedom of a region of space in are the same as that of a system of binary degrees of freedom distributed on the boundary of the region [6]. This point of view, represents an approach from a consistent theory of quantum gravity (unfortunately not yet found) in order to clarify the nature of dark energy. The Holographic principle says that the number of degrees of freedom of a physical system should scale with its bounding area rather than with its volume. Along these lines the literature have been focused in explain the size of the dark energy density on the basis of holographic ideas, derived from the suggestions that in quantum field theory a short distance a cut-off is related to a long distance cut-off due to the limit set by the formation of a black hole 7]. From the brane world approach the holographic principle was implemented in Ref. [8] where was studied the cosmological evolution of the holographic dark energy in a cyclic universe, generalizing the model of holographic dark energy proposed Ref. [9].

The plan of the paper is as follows: In Sec. II we specify the effective equation on the brane and give some generalities. In Sec. III we discuss a closed universe with an holographic density energy on the brane. Finally, we conclude in Sec. IV. 


\section{GENERALITIES}

We are going to consider an homogeneous and isotropic 4-brane described by the FLRW metric, in the case of closed universe where the gravitational sector of the field equations is described by a modified Friedmann equation given by (we adopt a unit system where $8 \pi G=1$ )

$$
3\left(H^{2}+\frac{1}{a^{2}}\right)=\rho\left(1 \pm \frac{\rho}{2 \lambda}\right),
$$

where $H=\dot{a} / a$ is the Hubble parameter with overdot stands for derivatives with respect to the cosmic time, and signs are related to positive and negative brane tension $(\lambda)$, respectively. This modified Friedmann equation can be derived from Randall Sundrumm model [1, 2] after to projected the five dimensional Einstein equations onto a four dimensional Friedmann brane [10] (this kind of modified Friedmann equation can be derived also from effective loop quantum cosmology [11]). If the matter fields are confined to the brane, they satisfy the standard conservation equation

$$
\dot{\rho}+3 H(\rho+p)=0
$$

From Eqs.(4) and (5) is straightforward to disguise the Friedmann equation in the standard form,

$$
\begin{aligned}
3\left(H^{2}+\frac{1}{a^{2}}\right) & =\rho_{e f f} \\
\dot{H} & =-\frac{1}{2}\left[\rho_{e f f}+p_{e f f}\right]+\frac{1}{a^{2}},
\end{aligned}
$$

where the effective quantities are given by

$$
\begin{aligned}
& \rho_{e f f}=2 \lambda x(1 \pm x), \\
& p_{e f f}=2 \lambda x[\omega(1 \pm 2 x) \pm x]
\end{aligned}
$$

and $x=\rho / 2 \lambda$ and $p=\omega \rho$. The effective equation is reads as follow,

$$
1+\omega_{e f f}(x)=-\frac{2}{3} \frac{\dot{H}-a^{-2}}{H^{2}+a^{-2}},
$$

and for the effective barotropic index we get,

$$
\omega_{e f f}=\frac{p_{e f f}}{\rho_{e f f}}=\frac{1}{1 \pm x}[(1 \pm 2 x) \omega \pm x],
$$

where the signs are related to positive and negative brane tension, respectively. Note that the inclusion of the brane in the theory change the role of the barotropic parameter $(\omega)$ by another effective parameter called $\omega_{\text {eff }}$. The equation (11) can be writes in the following way

$$
1+\omega_{e f f}(x)=\left(1 \pm \frac{x}{1 \pm x}\right)(1+\omega),
$$

then is clear that the presence of the brane implies that the quantity related to observational data is $\omega_{e f f}$ being $\omega$ a bare quantity.

It is interesting to consider the limit that possess Eq. 111) in the case of negative brane tension. When $x<<1$, we are recovering the standard Friedmann equation, and the barotropic index tend to a constant $\omega_{e f f}(x \rightarrow 0) \rightarrow \omega$, i.e one recovers the standard four dimensional general relativity. On the opposite limit when $x \rightarrow 1$ (strong limit) we obtain

$$
\omega_{e f f}(x \rightarrow 1) \rightarrow-\frac{\omega+1}{1-x},
$$

and it is possible to avoid the singularity if we take $\omega=-1$, then $\omega_{\text {eff }}(x)=-1$. 


\section{CLOSED UNIVERSE AND HOLOGRAPHY}

It is well established that in quantum field theory a short distances the UV cutoff is related to a long distance IR cutoff $(L)$ due to the limit set by forming a black hole [7]. This implies that the total energy of the system with size $L$ should not exceed the mass of the associated black hole. This fact traduce that the holographic density must be satisfied $L^{3} \rho_{H} \leq L M_{p}^{2}$. Thus the holographic energy density is chosen as the one that saturating this inequality and is given by

$$
\rho(L)=\frac{3 c^{2} M_{p}^{2}}{8 \pi L^{2}}
$$

where $M_{p}$ represent the Planck mass and $3 c^{2}$ is a numerical factor. We consider the Hubble parameter as our IR cutoff $\left(L=H^{-1}\right)$. Then the holographic energy density is given by

$$
\rho(H)=3 c^{2} H^{2},
$$

where we adopt natural units. If we consider a closed universe, from Eqs. (15) and (4) we obtain the following equation for the Hubble parameter

$$
\left(1-c^{2}\right) H^{2}= \pm \frac{1}{6 \lambda}\left(3 c^{2}\right)^{2} H^{4}-\frac{1}{a^{2}},
$$

this equation (note that, in the flat case Eq. (39) always drives to de Sitter expansion) gives different physical behavior depending if $c^{2}>1$ or $c^{2}<1$ (this occurs because we have two possible choices of the signs of $c^{2}$ and $\lambda$ ). In the following we focus on this point.

\section{A. Case $c^{2}<1$ and $\lambda>0$}

If $c^{2}<1$, the left side of Eq. (39) is positive and this implies that $\lambda>0$ (the option $c^{2}<1$ and $\lambda<0$ drives to a non-physical behavior, $H^{2}<0$ ), and this equation can be written in the following way

$$
\left(1-c^{2}\right) H^{2}=\frac{1}{6 \lambda}\left(3 c^{2}\right)^{2} H^{4}-\frac{1}{a^{2}} .
$$

The algebraic solution of (17) is

$$
H^{2}(a)=\alpha\left(1+\sqrt{1+\beta a^{-2}}\right)
$$

where the constants are given by

$$
\alpha=\frac{3 \lambda\left(1-c^{2}\right)}{\left(3 c^{2}\right)^{2}}, \beta=\frac{2}{3} \frac{\left(3 c^{2}\right)^{2}}{\lambda\left(1-c^{2}\right)^{2}} .
$$

Using (18) is straightforward to get

$$
\dot{H}(a)=-\frac{1}{2} \alpha \beta a^{-2} \frac{1}{\sqrt{1+\beta a^{-2}}} .
$$

With the definition of redshift $1+z=a_{0} / a$, the acceleration of the universe gets

$$
\dot{H}(z)+H^{2}(z)=\alpha\left[1+\frac{1+\frac{\beta}{2 a_{0}^{2}}(1+z)^{2}}{\sqrt{1+\frac{\beta}{a_{0}^{2}}(1+z)^{2}}}\right]>0,
$$

and now in according to Eq. (11) the effective barotropic index can be written as

$$
\omega_{e f f}(z)=\frac{\omega\left[1+\frac{\left(1-c^{2}\right)}{c^{2}}\left(1+\sqrt{1+\frac{\beta}{a_{0}^{2}}(1+z)^{2}}\right)\right]+\frac{\left(1-c^{2}\right)}{2 c^{2}}\left(1+\sqrt{1+\frac{\beta}{a_{0}^{2}}(1+z)^{2}}\right)}{1+\frac{\left(1-c^{2}\right)}{2 c^{2}}\left(1+\sqrt{1+\frac{\beta}{a_{0}^{2}}(1+z)^{2}}\right)},
$$


for early time, its limit limit behavior is

$$
1+\omega_{e f f}(z \rightarrow \infty) \rightarrow 2(1+\omega),
$$

and the respective limit for the acceleration

$$
\ddot{a}(t \rightarrow 0) \rightarrow \frac{\alpha \sqrt{\beta}}{2} \Rightarrow a(t) \sim t^{2} \Leftrightarrow \omega_{e f f}(z \rightarrow \infty) \rightarrow-\frac{2}{3} .
$$

Therefore, at early times we can say that the effective system associated with the presence of the brane and the holographic energy as main ingredients, is described by a membrane gas which has an effective equation of state given by $p_{\text {eff }} \rightarrow-\frac{2}{3} \rho_{\text {eff }}$, and the bare matter (associated with $\omega$ ) is doomed to becomes described by a component whose behavior is near to a cosmological constant $\omega \sim-1$ behavior, but it does not exactly correspond to cosmological constant.

And for late time

$$
1+\omega_{e f f}(z \rightarrow-1) \rightarrow\left(2-c^{2}\right)(1+\omega)
$$

and the respective limit for the acceleration

$$
\ddot{a}(t \rightarrow \infty) \rightarrow 2 \alpha a \Rightarrow a(t) \sim \exp (\sqrt{2 \alpha} t) \Leftrightarrow \omega_{\text {eff }}(z \rightarrow-1) \rightarrow-1,
$$

in this case the effective and the bare description are exactly described by a cosmological regimen. It is gives an accelerated evolution driven by a de Sitter expansion.

Then these scenarios where the presence of the brane is reflected in the fact that we have positive brane tension and the inclusion of holographic energy density allows to unify at least two accelerated phases in the evolution of the universe, described by a component whose behavior at early time is near cosmological constant and late time is exactly as a cosmological constant. Therefore the barotropic bare index $\omega$ has a slow variation in the neighborhood of cosmological constant domain.

\section{B. $c^{2}>1$ and $\lambda<0$}

Now if we consider that $c^{2}>1$ implies that $\lambda<0$ (the option $c^{2}>1$ and $\lambda>0$ drives to a non-physical behavior, $H^{2}<0$ ), the expression (17) is

$$
\left(c^{2}-1\right) H^{2}=\frac{1}{6 \lambda}\left(3 c^{2}\right)^{2} H^{4}+\frac{1}{a^{2}},
$$

and the solutions of this equation are

$$
H_{ \pm}^{2}(a)=\bar{\alpha}\left(1 \pm \sqrt{1-\bar{\beta} a^{-2}}\right)
$$

where the constant are given by

$$
\bar{\alpha}=\frac{3 \lambda\left(c^{2}-1\right)}{\left(3 c^{2}\right)^{2}} \text { and } \bar{\beta}=\frac{2}{3} \frac{\left(3 c^{2}\right)^{2}}{\lambda\left(c^{2}-1\right)^{2}}
$$

Such that, from Eq. (28) we obtain

$$
\dot{H}_{ \pm}(a)= \pm \frac{\bar{\alpha} \bar{\beta}}{2} \frac{1}{a^{2} \sqrt{1-\bar{\beta} a^{-2}}}
$$

and for the acceleration we get

$$
\dot{H}_{ \pm}(a)+H_{ \pm}^{2}(a)=\frac{\bar{\alpha}}{a^{2} \sqrt{1-\bar{\beta} a^{-2}}}\left[a^{2}\left(\sqrt{1-\bar{\beta} a^{-2}} \pm 1\right) \mp \frac{\bar{\beta}}{2}\right]
$$

From the positivity of the discriminant $1-\bar{\beta} a^{-2}$ we obtain the following constraint over the redshift

$$
\frac{a}{a_{0}}=(1+z)^{-1}>\left(1+z_{c}\right)^{-1}
$$


where the critical redshift value is given by

$$
1+z_{c}=\frac{a_{0}}{\sqrt{\bar{\beta}}}
$$

so the allowed redshift must be satisfy the following cosntraint

$$
-1 \leq z<z_{c}
$$

and we note that this constraint exclude the early times limit gives by $z>z_{c}$.

Now for the effective barotropic index we are applying a slightly different approach than the previous section. As the critical redshift does not allow to take at early times we do not write the effective index in terms of the bare barotropic index and we just focus our attention in effective description

$$
1+\omega_{e f f}^{ \pm}(z)=\mp \frac{1}{3} \Delta^{ \pm}(z)
$$

where

$$
\Delta^{ \pm}(z)=\left(\frac{1+z}{1+z_{c}}\right)^{2}\left[\sqrt{1-\left(\frac{1+z}{1+z_{c}}\right)^{2}}\left(1 \pm \sqrt{1-\left(\frac{1+z}{1+z_{c}}\right)^{2}}\right]^{-1}\right.
$$

such that, $\Delta^{ \pm}(z)>0$. From Eqs. (35) and (36) we obtain two possible schemes

$$
\omega_{e f f}^{+}(z)=-1-\frac{1}{3} \Delta^{+}(z)<-1,
$$

this corresponds to a phantom behavior that asymptotically goes to a genuine de Sitter expansion because $\Delta^{ \pm}(z \rightarrow-1) \rightarrow 0$. From the early limit $\left(z \rightarrow z_{c}\right)$, we observe a peculiar behavior of our physical quantities because appears an early singularity, that in the literature of phantom cosmology is classified as a sudden singularity, are described by $a$ and $\rho$ finite and $|p|$ infinite [12].

$$
\omega_{e f f}^{-}(z)=-1+\frac{1}{3} \Delta^{-}(z)
$$

that represent a transitory behavior starting with matter, passing to a quintaessential transitory phase and an asymptotically like cosmological constant $\omega_{e f f}^{-} \rightarrow-1$ behaviors. Although $\omega_{e f f}^{-} \rightarrow-1$ the evolution of scalar factor does

not goes as de Sitter expansion $\left(\ddot{a} \sim a^{-1}, z \rightarrow-1\right)$. Also this branch have a sudden early singularity but that does not correspond to a phantom singularity.

Summarize in a scenario where the brane tension is negative and the holographic parameter satisfies $c^{2}>1$, we obtain two branches that driven to different cosmological stages. First at all, we obtain a critical value over the redshift $\left(z_{c}\right)$, that constraint the physical region of our branches, where one of them represent an early phantom evolution goes asymptotically to a genuine de Sitter and the other one correspond to a early matter stage, then a transitory quintaessential phase and ending in an asymptotically like cosmological constant behavior. Also we want to note that both branches beginning with a early sudden singularity, where one of them correspond to a phantom singularity and the other does not generate a phantom. The difference lies in the early limit of the effective barotropic index because $\omega_{\text {eff }}^{ \pm} \rightarrow \mp \infty$.

\section{DISCUSSION AND OUTLOOK}

In this article we have studied a closed universe which a holographic energy on the brane whose energy density is described by $\rho(H)=3 c^{2} H^{2}$ and we obtain an equation for the Hubble parameter, given by

$$
\left(1-c^{2}\right) H^{2}= \pm \frac{1}{6 \lambda}\left(3 c^{2}\right)^{2} H^{4}-\frac{1}{a^{2}},
$$

this equation gave different physical behavior depending if $c^{2}>1$ or $c^{2}<1$ against of the sign of the brane tension. First we focus on the case $c^{2}<1$ and $\lambda>0$, where the presence of the brane is reflected in the fact that we have positive brane tension and the inclusion of holographic energy density allow to unify at least two accelerated phases in 
the evolution of the universe, described by a component whose behavior at early time is near cosmological constant and late time is a cosmological constant. Therefore the barotropic bare index $\omega$ has a slow variation in the neighborhood of cosmological constant domain. In the other case $c^{2}>1$ and $\lambda<0$ where the brane tension is negative and the holographic parameter that satisfies $c^{2}>1$, we obtain two branches that driven to different cosmological stages. First at all, we obtain a critical value over the redshift $\left(z_{c}\right)$, that constraint the physical region of our branches, where one of them represent an early phantom evolution goes asymptotically to a genuine de Sitter and the other one correspond to a early matter stage, then a transitory quintaessential phase and ending in an asymptotically like cosmological constant behavior. Also we want to note that both branches beginning with a early sudden singularity, where one of them correspond to a phantom singularity and the other does not generate a phantom. The difference lies in the early limit of the effective barotropic index because $\omega_{\text {eff }}^{ \pm} \rightarrow \mp \infty$. Therefore the inclusion of the brane world and holographic energy on the brane allow to obtain physical results when we choose the IR cutoff as the size of our universe $(L=1 / H)$. We hope to discuss in the near future the inclusion of the other two possibilities for $L[9]$.

\section{Acknowledgments}

This work was supported by COMISION NACIONAL DE CIENCIAS Y TECNOLOGIA through FONDECYT Grant 11060515 (JS). This work was also partially supported by PUCV Grants No. 123.701/2008 (SL), No. 123.789/2007 (JS) and by Dirección de Estudios Avanzados PUCV. Also was supported from DIUFRO DI08-0041 of Dirección de Investigación y Desarrollo Universidad de la Frontera (FP) . The authors SL and JS wish to thank Departamento de Física de la Universidad de La Frontera for its kind hospitality.

\section{References}

[1] L. Randall and R. Sundrum, 1999 Phys. Rev. Lett. 83, 3370.

[2] L. Randall and R. Sundrum, 1999 . Rev. Lett. 83, 4690.

[3] G. Gabadadze, 2007 Nucl. Phys. Proc. Suppl. 171, 88.

[4] P. Horava and E. Witten, 1996 Nucl.Phys.B 475, 94 ; P. Horava and E. Witten, 1996 Nucl.Phys.B $460,506$.

[5] R. Maartens, 2004 Living Rev. Rel. 7, 7.

[6] L. Susskind, 1995 J. Math. Phys. 36, 6377.

[7] A. G. Cohen, D. B. Kaplan and A. E. Nelson, 1999 Phys. Rev. Lett. 82, 4971.

[8] J. f. Zhang, X. Zhang and H. y. Liu, 2007 Eur. Phys. J. C 52, 693.

[9] M. Li, 2004, Phys. Lett. B 603, 1.

[10] T. Shiromizu, K. i. Maeda and M. Sasaki, 2000 Phys. Rev. D 62, 024012.

[11] M. Bojowald, 2005 Living Rev. Rel. 8, 11; A. Ashtekar, arXiv:gr-qc/0702030

[12] S. Nojiri, S. D. Odintsov and S. Tsujikawa, 2005 Phys. Rev. D 71, 063004. 